\title{
A Review on Various Formulation Methods in preparing Colon targeted mini-tablets for Chronotherapy
}

\author{
Mohd Abdul Hadi" ${ }^{*}$, N. G. Raghavendra Rao ${ }^{2}$, A. Srinivasa Rao ${ }^{3}$, Tayyaba Mahtab ${ }^{4}$, Sayeeda Tabassum ${ }^{4}$ \\ 'Department of Pharmaceutics, Bhaskar Pharmacy College, Yenkapally (V), Moinabad (M), R. R. District, Hyderabad-500075, Telangana, India. \\ 2Department of Pharmaceutics, Sree Chaitanya Institute of Pharmaceutical Sciences, LMD Colony, Thimmapur, Karimnagar-505001, Telangana, India. \\ ${ }^{3}$ Bhaskar Pharmacy College, Yenkapally (V), Moinabad (M), R. R. District, Hyderabad-500075, Telangana, India. \\ ${ }^{4}$ Department of Pharmaceutical Analysis, Bhaskar Pharmacy College, Yenkapally (V), Moinabad (M), R. R. District, Hyderabad-500075, Telangana, India.
}

\begin{tabular}{l}
\hline ARTICLE INFO \\
\hline Article history: \\
Received on: 21/12/2017 \\
Accepted on: 31/01/2018 \\
Available online: 30/03/2018 \\
\hline Key words: \\
Core-mini-tablets filled \\
pulsincap drug delivery \\
system, Matrix-mini-tablets \\
filled capsule drug delivery \\
system, Coated-mini-tablets \\
filled capsule drug delivery \\
system.
\end{tabular}

\begin{abstract}
Rheumatoid arthritis disease, according to its circadian rhythms shows early morning peak symptoms. Sometimes, single unit (ex. larger tablets) and multiple units (ex. granules, pellets) colon targeted drug delivery systems are not always an efficient treatment option. Because single unit larger tablets may possess the disadvantages of unintentional disintegration of the formulation due to GI variation or manufacturing deficiency leading to complete dose dumping. Even the multiple unit drug delivery systems such as granules and pellets also have many drawbacks because of their irregular weights, shapes and sizes. Thus, a tight, reproducible in-vitro and in-vivo release profile can't be achieved. In an attempt to overcome the problems presented by these delivery systems, advanced system such as multiple unit minitablets have developed. This is an approach towards achieving critical factors such as better patient compliance and convenience. In the present review, a concerted try has made to summarize the details of different formulation methods used in preparing mini-tablets of lornoxicam and naproxen drugs for colon targeted delivery in chronotherapy. The techniques formulated and evaluated as core-mini-tablets filled pulsincap drug delivery system (using time dependent polymers), matrix-mini-tablets filled capsule drug delivery system (using microsomal enzyme dependent and $\mathrm{pH}$ dependent polymers) and coated-mini-tablets filled capsule drug delivery system (using $\mathrm{pH}$ dependent polymers). All these methods were successful in targeting Anti-inflammatory drugs at colonic junction. Hence, the mentioned formulation methods can be successfully used in the chronotherapeutic treatment of Rheumatoid arthritis.
\end{abstract}

\section{INTRODUCTION}

Drugs have become the order of day for many people across the world. There is hardly anyone who has not taken a medicine in his/her life, but many times the people who take tablets rarely give importance to the timing of its intake. There are about 60 diseases including Arthritis, Asthma and Cancer for which drugs can be more effective when they are taken at the right time of the day. Because, ideal therapy results only when right portion of the drug is delivered to the right targeted organ at the most suitable time. Thus, many adverse effects can also be

${ }^{*}$ Corresponding Author

Mohd Abdul Hadi, M. Pharm., Ph.D, Associate Professor, Department of

Pharmaceutics, Bhaskar Pharmacy College, Yenkapally (V), Moinabad

(M), R. R. District, Hyderabad-500075, Telangana, India.

E-mail:hadi.lcp@gmail.com reduced when a drug is not given when actually it is not required (Hrashesky, 1994; Drelaywaja, 2010; Smolensky and Peppar, 2007; Suresh and Pathak, 2005).

Further, the biochemistry and physiology of a human being is not constant during the 24 hour period, but varies according to the peak timing and trough of body's circadian processes and functions. Many human body systems such as pulmonary, cardiovascular, hepatic and renal vary in their functions throughout a day. It has become obvious through clinical and epidemiological studies that even the disease activity levels of a number of disorders such as Asthma, Arthritis, Peptic ulcer, Hypertension, etc., have a pattern related to body's biological clock as stated to circadian rhythms. When the normal biological processes are influenced according to the time of the day, they affect the pathophysiology of the disease and its treatment. Thus, many human physiological processes differ in a rhythmic manner 
and are affected by the sleep wake cycle in synchrony with the internal biological clock (Smolensky and Peppar, 2007; Suresh and Pathak, 2005).

The association of biological rhythms of the human body to conventional environmental cycles has been known for centuries. The study of biological rhythms like tidal, yearly, seasonally, weekly and daily rhythms and their underlying mechanisms is called chronobiology (Suresh and Pathak, 2005). This idea was introduced into clinical medicine in the 1950s for the prevention of diseases. Also, many ancient healers have reported that for the treatment to be successful and effective it has to be given according to various internal and external cycles. Coordinating medical treatment with biological rhythms is called chronotherapy, whereas the study related with the drug delivery according to inherent disease activities for a certain time period is called chronotherapeutics. The possible advantages of chronotherapeutics have been evidenced in the control of a number of diseases. By using this science, can help in early diagnosis, prevention and treatment of diseases and thus a reduction in the overall healthcare costs. The main purpose of chronotherapy in the treatment of various diseases is to release the highest drug concentration during the time of its greatest need as per the circadian onset of disease. For example, Rheumatoid arthritis disease can be effectively treated by utilizing the chronotherapy concept for maintaining the highest drug concentration in the systemic circulation during the early morning time. So that peak stiffness and pain of this disease can be controlled (Drelaywaja, 2010; Shivkumar et al., 2007; Sajan et al., 2009).

The medication according to chronotherapy, can be effected by the judicious timing of capsules and tablets. Sometimes, it requires a modified drug delivery technology to match concentrations of drug to rhythms in the disease activity (Hrashesky, 1994; Suresh and Pathak, 2005; Smolensky and Peppar, 2007; Drelaywaja, 2010). In such cases, intentionally delaying the absorption or colonic targeting of drugs can be beneficial so as to have a uniform therapeutic effect. Hence, the drug releases after a lag time and will be available in highest concentration during the specific time of its greatest need. Thus, better therapeutic effectiveness and uniform patient compliance can be easily achieved by colon targeted drug delivery in chronotherapy (Gothaskar and Joshi, 2004; Krishainah and Satyanarayana, 2001).

The oral route of drug administration is recognized as the most widely accepted route because of its benefits of compactness, self-administration and easy manufacturing. However, it is predictable that at least ninety percentage of all drugs which are used to produce therapeutic effect are administered through oral route (Lachman, Lieberman, 2009). Oral drug delivery systems can be divided in two broader groups such as single unit dosage forms (SUDFs ex. tablets) and multiple unit dosage forms (MUDFs ex. pellets, granules and mini-tablets) (Carla et al., 2006). Single and multiple unit drug delivery systems are not always an efficient treatment option due to their drawbacks. Because SUDFs such as larger tablets, may possess the problems of unintentional disintegration due to unusual gastric physiology or manufacturing deficiency which may lead to excessively compromised systemic bioavailability of drug or even loss of its local therapeutic action (Laila et al., 2006). Moreover, even the MUDFs such as granules and pellets also have a lot of drawbacks due to their irregular weights, shapes and sizes. Thus a tight, accurate and reproducible release profile can't be achieved, which is very important in colon targeted drug delivery systems as well as in Chronotherapy.

As mentioned, single unit larger tablets even though they have uniform shapes, sizes and weights, but still there are more chances of dose dumping, systemic toxicity, decreased systemic drug bioavailability or even loss of its local therapeutic action. Moreover, in granules and pellets the chances of dose dumping and systemic toxicity is less, but still they are not uniform in sizes, shapes and weights which lead to failure in achieving drug content uniformity and reproducible release profiles. In an attempt, to overcome the problems presented by single unit (ex. larger tablet) and multiple unit (ex. granules, pellets) colon targeted drug delivery systems, an improved dosage form is required which releases the drug at a uniform and pre-determined rate to ensure patient compliance. Thus, innovative thinking has transformed towards the concept of multiple unit mini-tablets for colon targeted drug delivery in chronotherapy. Because, these mini-tablets possess the combined advantages of a single unit larger tablet and multiple unit granules and pellets, but still devoid of the chances of dose dumping, systemic toxicity, variation in formulation behavior and drug release profile due to unit to unit variation and within the unit variation.

Lornoxicam has potent anti-inflammatory and analgesic properties and has been generally used for the associated treatment of inflammation and pain in Rheumatoid arthritis (Mohd et al., 2014; Mohd et al., 2015). Also, naproxen is effective in both experimental and clinical pain of Rheumatoid arthritis and it is a naphthylpropionic acid derivative (Hadi et al., 2014; Mohd et al., 2016). They both belong to the class of NSAID's.

\section{RATIONALE FOR DEVELOPING MINI-TABLETS AS DRUG DELIVERY SYSTEM}

Mini-tablets are smaller tablets having diameter equal to or less than $3 \mathrm{~mm}$ that can be either filled into a capsule shell or placed in sachets for easy administration. They are also known as mini-matrices, and can be used as multiparticulate dosage forms. They combine the physiological benefits of multiple unit dosage forms (ex. Granules, pellets, microparticles etc.) with economic benefits of single unit dosage forms (ex. Bigger tablet). Thus, these mini-tablets are considered as a promising patient friendly drug delivery system (Aleksandar et al., 2015; Sona et al., 2015; Mirelabodea et al., 2010).

Mini-tablets have several advantages as an oral drug delivery system and these include the following:

1 . They can be easily manufactured with simple techniques of tableting as a result of which several complex steps, time and losses in manufacturing can be reduced and thus can be produced in a continuous and reproducible way. Gaber et al. (2015) reported that as per the industrial aspects, the time taken for compressing $5 \mathrm{~kg}$ of powder for mini-tablets were notably shorter (i.e. 1 hour) while compared to that required for preparing the pellets of the same amount (i.e. 4 hour). Also, they reported that the loss due to dust while compressing the mini-tablets was only $2.3 \%$ in comparison to $20-30 \%$ for pellets (Schmidt et al., 1999; Motor et al., 2014; Gaber et al., 2015). 
2. In a study, it was claimed by Lennartz and Mielck that higher amounts of active ingredients can be easily manufactured or tabletted in mini-tablets compared to larger tablets. They also offer a broad range of drug release rate patterns and adjustment of these release rates. They have also reported reduced capping and greater mechanical strength for mini-tablets in comparison to conventional larger tablets when they both are produced at a same higher pressure. They concluded by noting the ratio difference between the outer surface and volume of tablet that because of the smaller diameter of mini-tablets this ratio increases. Thus, because of these results it makes mini-tablets dosage forms as more attractive, for drugs which are known to create more problems during manufacturing (Lennartz and Mielck, 1998).

3 . They can be prepared by either direct compression or wet granulation method using the conventional rotary compression equipment which is fitted with multi-tip punches. For individual dosing, they can be administered using a dose dispenser or for applying, the compacts of dosage can be easily filled into hard gelatin capsules as similar to other multiparticulates. While for dosing the multiparticulates, standard volumetric dosing systems has to be used and for mini-tablets they can easily count using special counting/feeding stations. Therefore in mini-tablets, the chances of poor dosing accuracy due to volumetric systems can be reduced and thus there will be very less variation among the formulation units (Schmidt et al., 1999; Motor et al., 2014; Gaber et al., 2015; Lennartz and Mielck, 1998; Deepak et al., 2013; Karthikeyan, 2013).

4. Their defined size, shape, weight, constant specific surface area, smooth outer surface, robust mechanical properties, small variability within and between the batches contributes to the more uniform reproducible coating by using less coating material in comparison to granules or pellets. Gaber et al. (2015), reported that the mini-tablets showed extended Venlafaxine hydrochloride release i.e. $<60 \%$ in 8 hrs which was equally comparable to the Effexor XR pellets. Remarkably, the formulated pellets required higher coating thickness to provide similar retardation effect for drug release (Gaber et al., 2015; Sirisha, 2012; Hue et al., 2007).

5. They are having less stability problems as they don't need solvents for their production. A study was conducted by Gaber et al. (2015), by subjecting the prepared coated minitablets and pellets with $6 \%$ and $15 \%$ weight gain respectively to shelf stability studies for a period of 6 months. The results have shown remarkable changes in drug release profile for pellets, whereas the release profile for mini-tablets was found to be intact. Thus, it was reported that mini-tablets have higher stability when compared with pellets (Gaber et al., 2015).

6. Similar to the other multiparticulates the mini-tablets filled capsules can also be used as a sprinkle formulation because of which high degree of dispersion in the GIT can be maintained and thus they minimize the risks of high local drug concentrations, dose dumping and systemic toxicity (Dey et al., 2008; Ivanovska, 2014; Anne et al., 2013; Patel and Patel, 2010).

7. When compared to single unit formulations, they decreases the irritation effect in the GIT for local irritating drugs as the dose is divided in between multiple unit mini- tablets. Thus, they can be more useful for GI irritant drugs such as NSAIDs (Patel and Patel, 2010).

8. As shown by a number of bio-studies which were performed during development work, clearly proves that they are having minimal effect of food on plasma drug release profile in the GIT and can be administered irrespective of the fasting or fed state. They can act separately from the food transport rhythms and reduces intra and inter-subject variability by maintaining smooth sustained plasma levels. Thus, reliability, reproducibility and predictability of the dosage form can be easily achieved which leads to increased patient compliance (Jain et al., 2009).

By observing the advantages, it is proved that minitablets production on a large scale can be considered as time and money saving as they offers simple production procedures, less wastage of materials, accurate dosing, good mechanical strength, less coating material, good stability, higher drug loading capacity and yield. Thus, from the industrial point of view, mini-tablets are superior to all the other multiple unit dosage forms in terms of product quality, manufacturing and economical aspects. This technology can be of absolute importance and also a benefitable investment for pharmaceutical industries, mainly in the developing countries (Gaber et al., 2015).

In the present review, the reported formulation methods in preparing colon targeted mini-tablets have been summarized. The aim is to present various formulation methods in preparing mini-tablets for colonic targeting of Anti-inflammatory drugs in the chronotherapeutic treatment of Rheumatoid arthritis.

\section{CORE-MINI-TABLETS (CRMT) FILLED PULSINCAP FORMULATIONS OF LORNOXICAM}

The CRMT filled pulsincap formulations of lornoxicam were prepared by following the 4 below mentioned steps.

\section{Step 1-Preparation of cross-linking gelatin capsules}

At first, formalin vapors were developed by adding a pinch of potassium permanganate $\left(\mathrm{KMNO}_{4}\right)$ in a desiccator filled with $25 \mathrm{ml}$ of $15 \% \mathrm{v} / \mathrm{v}$ solution of formaldehyde. Then a screen consisting of about 100 empty hard gelatin capsule bodies was exposed to the generated vapors of formaldehyde. Whereas the caps were not treated with formaldehyde vapors to keep them water soluble. This desiccator was then tightly closed with a lid. In order to confirm that the capsule bodies thoroughly react with the generated formaldehyde vapors, the desiccator was left aside for a time period of 12 hours. Finally, capsule bodies were withdrawn from the desiccator and dried at $50^{\circ} \mathrm{C}$ for a time period of 30 minutes and after capping with untreated caps were placed in polythene bags (Mohd et al., 2015; Mastiholimath et al., 2007; Sindhu et al., 2007; Meena et al., 2011).

\section{Step 2-Preparation of polymer plugs}

The hydrophilic polymer plugs which are used for closing the open end of capsule bodies were developed using direct compression method. The plugs were prepared by compressing polymer (Sodium carboxy methyl cellulose or HPMC-K100M or Sodium alginate) and lactose using $6 \mathrm{~mm}$ dies and punches on a rotary tablet press (by keeping constant weight, hardness and thickness values for all the hydrogel plugs) (Mohd et al., 2015; 
Mastiholimath et al., 2007; Sindhu et al., 2007; Meena et al., 2011).

\section{Step 3-Preparation of CRMT}

All the ingredients (i.e. Lornoxicam, PVP K-30, Avicel PH 102) were passed through a 100 size sieve, and after weighing as per the required quantity there were finally blended. The prepared mixture after lubricating with magnesium stearate and aerosil was compressed using direct compression method, to a weight of 25 $\mathrm{mg}$ for each mini-tablet using $3 \mathrm{~mm}$ round concave punches in a rotary tablet press (Rimek mini-press, M/s Karnavathi engineering Ltd., Ahmadabad) (Mohd et al., 2014; Mohd et al., 2015; Hadi et al., 2014; Mohd et al., 2016).

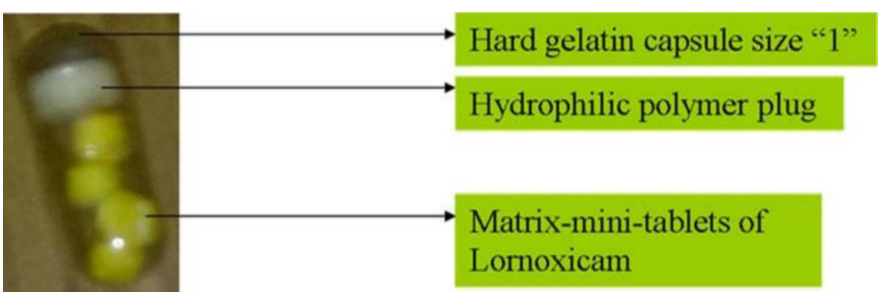

Fig. 1: Core-mini-tablets filled pulsincap formulation of lornoxicam.

\section{Step 4-Development of Pulsincap dosage form of lornoxicam}

The final CRMT filled pulsincap formulation as shown in Figure 1 was developed by filling four CRMT equivalent to eight milligrams of lornoxicam into the formaldehyde exposed hard gelatin capsule bodies. The open end of capsule bodies was closed by plugging it with different polymers like sodium carboxy methylcellulose or HPMCK100M or sodium alginate separately at different concentrations $(20,30$ and $40 \mathrm{mg})$. A small amount of $5 \%$ ethanolic solution of ethylcellulose was used to stick the joint between the cap and capsule body. These sealed capsules, were then coated with a $5 \% \mathrm{w} / \mathrm{v}$ cellulose acetate phthalate in $8: 2$ $\mathrm{v} / \mathrm{v}$ solution of acetone: ethanol which was then plasticized with $0.75 \%$ of dibutylphthalate. The above coating of the capsules was done by dip coating method with an aim to prevent variable gastric emptying. The coating was continued until 4 to $6 \%$ increase in weight of the complete pulsincap formulation was obtained. After coating, percentage weight gain in the capsules was determined (Mohd et al., 2015; Mastiholimath et al., 2007; Sindhu et al., 2007; Meena et al., 2011).

\section{In-vitro dissolution testing observations of CRMT filled pulsincap formulations of lornoxicam}

While performing the dissolution testing for all the CRMT filled pulsincap formulations, it was found that as the individual concentrations of HPMC-K100M, sodium alginate, sodium carboxymethylcellulose in polymer plugs were increasing, the lag time and the release rate of lornoxicam in mini-tablets was found to be increased and decreased respectively. It is due to the reason that the plugged hydrophilic polymer after continuously absorbing the surrounded dissolution medium has swelled and thus formed a swollen matrix which after completely wetting became a soft mass and thus came out of the capsule body (Mohd et al.,
2015; Mastiholimath et al., 2007; Sindhu et al., 2007; Meena et al., 2011). Whereas, it was also found that optimized CRMT filled pulsincap formulation can be prepared by filling four CRMT into a size " 1 " HPMC capsule and plugging with $30 \%$ concentration of HPMC K100M polymer and further coating the entire capsule with 5\% cellulose acetate phthalate (Mohd et al., 2015).

\section{MATRIX-MINI-TABLETS (MAMT) FILLED CAPSULE FORMULATIONS OF LORNOXICAMAND NAPROXEN}

The MAMT of lornoxicam and naproxen were formulated using miscrosomal enzyme dependent (Guar gum and Sodium alginate) and $\mathrm{pH}$ dependent (Eudragit L100 and Eudragit S100) polymers in both individual and combined concentrations (10$60 \%$ ) by direct compression technique. A total of 36 formulations were prepared for lornoxicam and 24 formulations were prepared for naproxen depending upon the doses of drugs. For preparation, initially drug (Lornoxicam/Naproxen), polymers and avicel $\mathrm{PH}$ 102 were allowed to pass through a 60 mesh sieve, then weighed and mixed. Similarly, aerosil and magnesium stearate were also passed separately through the same sieve and after weighing were added to the above mixture and thoroughly blended. This final prepared blend was then compressed into $25 \mathrm{mg}$ mini-tablets by using $3 \mathrm{~mm}$ round concave punches in a rotary tablet press (Rimek mini-press, M/s Karnavathi engineering Ltd, Ahmadabad). For preparing MAMT filled capsule formulation of lornoxicam, four MAMT equivalent to $8 \mathrm{mg}$ of lornoxicam were filled into a size ' 4 ' HPMC capsule as shown in Figure 2 (Carla et al., 2006; Mohd et al., 2014; Noorana et al., 2013; Brabander et al., 2000; Mohapatra et al., 2011). Whereas, for preparing MAMT filled formulation of naproxen, fifteen MAMT equivalent to $250 \mathrm{mg}$ of naproxen into were filled into a size '0' HPMC capsule as shown in Figure 3 (Carla et al., 2006; Mohd et al., 2016; Noorana et al., 2013; Brabander et al., 2000; Mohapatra et al., 2011).

\section{In-vitro dissolution testing observations of MAMT filled capsule formulations of lornoxicam and naproxen}

While performing the dissolution testing for all the MAMT filled capsule formulations of lornoxicam and naproxen, it was found that as the individual concentrations of sodium alginate, guar gum, eudragit L100 and eudragit S100 were increasing in mini-tablets, the lag time and the release rate of lornoxicam was found to be increased and decreased respectively. For mini-tablets prepared with sodium alginate, it is due to the reason that the hydrophilic polymer after continuously absorbing the surrounded dissolution medium, has completely been swelled and thus formed a swollen matrix and delayed the drug release. Whereas for mini-tablets prepared with guar gum polymer, it is due to the characteristics of enzyme dependent polysachcharide polymers, just because of which it maintained insolubility in the stomach and small intestine and released the drug only in the availability of colonic enzymes because of their fermentation. And for mini-tablets prepared with Eudragit L100 and Eudragit $\mathrm{S} 100$ polymers, it is because of their $\mathrm{pH}$ dependent solubilities in $\mathrm{pH} 6.0$ and $\mathrm{pH} 7.0$ buffers respectively. When the combination of guar gum, sodium alginate and Eudragit L100 and Eudragit S100 polymers were used, it was found that the release rate of the used Anti-inflammatory drugs was increased comparing to mini-tablets prepared with guar gum and Eudragit S100 alone. In 
guar gum and sodium alginate combination, it is due to the quickly water absorbing tendency of sodium alginate polymer which has increased the drugs permeation from guar gum polymer. Whereas in Eudragit L100 and Eudragit S100 polymers combination, it is due to Eudragit L100 polymer because of its good solubility in $\mathrm{pH}$ 6.5 and 6.8 buffers which has increased the permeation of drugs from Eudragit S100 polymer as it dissolves at $\mathrm{pH} 7.2$ buffer.

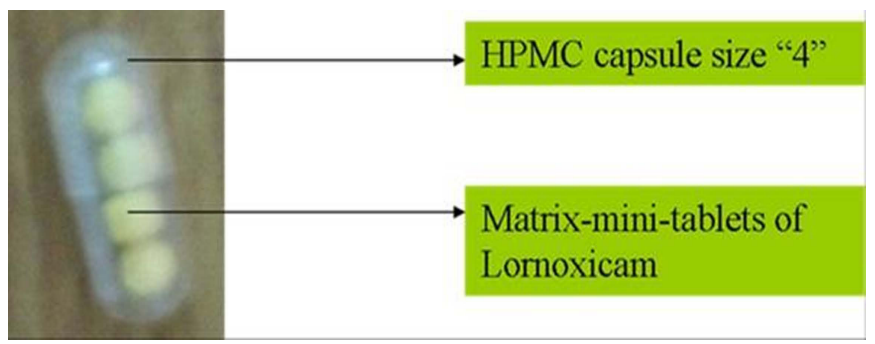

Fig. 2: Matrix-mini-tablets filled capsule formulation of lornoxicam.

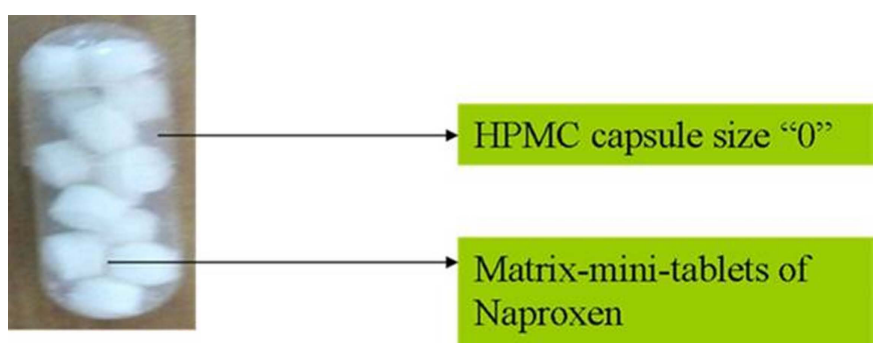

Fig. 3: Matrix-mini-tablets filled capsule formulation of naproxen.

Whereas, it was also found that the optimized MAMT filled capsule formulation of lornoxicam can be prepared by filling four matrix-mini-tablets (formulated with 40\% combined concentrations of Eudragit L100 and Eudragit S100 polymers in 1:3 ratio) into a size "4" HPMC capsule. Moreover, the optimized MAMT filled capsule formulation of naproxen can be prepared by filling fifteen matrix-mini-tablets (formulated with $32 \%$ concentration of Eudragit S100 polymer) into a size "0" HPMC capsule (Mohd et al., 2014; Mohd et al., 2016).

\section{In-vivo and In Vitro-In Vivo Correlation observations of MAMT filled capsule formulation}

The optimized MAMT filled capsule formulation of lornoxicam was successfully determined for its pharmacokinetic parameters and in vitro-in vivo correlation. The pharmacokinetic finding results clearly indicate that lornoxicam formulated as MAMT filled in a capsule may be considered as a novel dosage form for colonic targeted delivery. The in vitro-in vivo correlation result also suggests an appreciable linear relationship between the lornoxicam fraction released and the lornoxicam fraction absorbed. Hence, the developed MAMT filled capsule formulation can be used in the chronotherapeutic treatment of rheumatoid arthritis (Abdul et al., 2014).

\section{COATED-MINI-TABLETS (COMT) FILLED CAPSULE FORMULATIONS OF NAPROXEN}

The COMT of Naproxen was prepared using the wet granulation method. For preparation, initially naproxen, the intragranular portion of sodium starch glycollate, PVP-K30 and lactose were separately passed through a 60 mesh sieve and were mixed in dry condition. Then, dry mixing of blend was done at a slow speed in a blender for a time period of 10 minutes, followed by granulating with ethanol. This prepared wet granulated mass was immediately passed through a 16 mesh size sieve. The obtained sieved granules were completely dried in a hot air oven at $30-35^{\circ} \mathrm{C}$ for a time period of 1 hour. Then, after drying, they were allowed to pass through the same sieve so as to break the irregular shaped agglomerates and mixed with an extragranular portion of sodium starch glycollate. Finally, these granules after proper lubrication with magnesium stearate and aerosil were compressed into $25 \mathrm{mg}$ mini-tablets using $3 \mathrm{~mm}$ round concave punches in a rotary tablet press (Rimek mini-press, M/s Karnavathi engineering Ltd, Ahmadabad).

The coating solution was prepared by mixing the eudragit polymers in $50 \%$ of all the solvents (acetone, isopropyl and water) and stirring them for a time period of 60 minutes till completely dissolving of both the polymers. Thereafter, triethyl citrate and talc were also added in the remained solvent mixture and were stirred with a high speed mixer for a time period of 10 minutes. Finally, the triethyl citrate and talc solution were slowly poured into the eudragit solution in the stirring mode and was allowed to pass through a $0.5 \mathrm{~mm}$ sieve.

Then prepared mini-tablets were coated along with placebo tablets using $500 \mathrm{ml}$ of coating solution by varying the ratios $(1: 0,2: 1,1: 1,1: 2,0: 1)$ and coating level $(10,15,20 \%)$ of eudragit L100 and Eudragit S100 polymers in a ' 6 ' inches lab coating pan using a spray gun (United Technologies, Mumbai, India). The operating parameters were-tablets charge 100 grams; pre-heating temperature $40 \pm 5^{\circ} \mathrm{C}$; pre-heating time 20 minutes; inlet air temperature $45 \pm 5^{\circ} \mathrm{C}$; diameter of nozzle $1.0 \mathrm{~mm}$; atomization pressure 2.0 bar; spray rate $8-10 \mathrm{ml} / \mathrm{min}$.

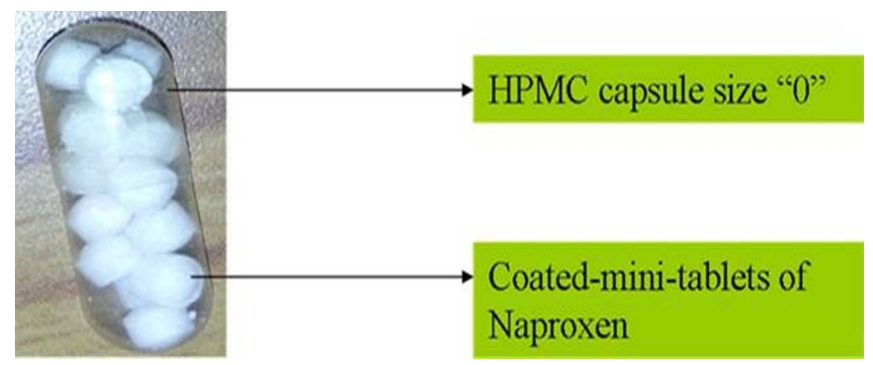

Fig. 4: Coated-mini-tablets filled capsule formulation of naproxen.

For preparing the capsule formulation, fifteen enteric coated COMT equivalent to $250 \mathrm{mg}$ of naproxen was filled in size '1' HPMC capsule as shown in Figure 4 (Hadi et al., 2014; Shivkumar et al., 2007; Akhgari et al., 2005; Evans et al., 1988).

\section{In-vitro dissolution testing observations of COMT filled capsule formulations}

While performing the dissolution testing for all the COMT filled capsule formulations, it was found that as the individual concentrations of eudragit L100 and eudragit S100 polymers in mini-tablets were increasing the lag time and release rate of naproxen was found to be increased and decreased respectively. It is due to $\mathrm{pH}$ dependent solubilities of Eudragit 
L100 and Eudragit S100 polymers coating in $\mathrm{pH} 6.0$ and $\mathrm{pH} 7.0$ buffers respectively. Whereas in eudragit L100 and eudragit S100 polymers combination coating, it is due to the good solubility of eudragit L100 polymer in $\mathrm{pH} 6.5$ and 6.8 buffers which has increased the permeation of drug from Eudragit S100 polymer as it dissolves at pH 7.2 buffer (Hadi et al., 2014; Shivkumar et al., 2007; Akhgari et al., 2005; Evans et al., 1988).

\section{In-vivo and In Vitro-In Vivo Correlation observations of COMT filled capsule formulations}

The optimized COMT filled capsule formulation of naproxen was successfully determined for its pharmacokinetic parameters and in vitro-in vivo correlation. The pharmacokinetic finding results clearly indicate that naproxen formulated as COMT filled in a capsule may be considered as a novel dosage form for ileocolonic targeted delivery. The in vitro-in vivo correlation result also suggests an appreciable linear relationship between the naproxen fraction released and the naproxen fraction absorbed. Hence, the developed COMT filled capsule formulation can be used in the chronotherapeutic treatment of rheumatoid arthritis (Hadi et al., 2015).

\section{CONCLUSION}

The various research works mentioned in this review, clearly suggests that the time, $\mathrm{pH}$, and microsomal enzyme dependent polymers can be suitable for preparing mini-tablet formulations for colon targeted delivery of Anti-inflammatory drugs. This approach has been utilized in the chronotherapeutic treatment of Rheumatoid Arthritis. The obtained optimized formulae can also be explored for various other categories of drugs that can be used for chronotherapy. The developed optimized formulations of this review using mini-tablets technology combine the physiological advantages of multiple unit dosage forms (ex. granules, pellets, microparticles etc.) with economic benefits of single unit dosage forms (ex. bigger tablet) and also has a number of advantages from the industrial point of view. This technology can be of profitable investment and also the ultimate importance for Pharmaceutical Industries especially in the developing countries.

\section{ACKNOWLEDGMENT}

The authors are very much thankful to the Secretary of JB group of Educational Institutions Sri. J. Bhaskar Rao garu for his constant help, support and encouragement to the academics generally and research particularly. The authors are also thankful to him for providing suitable research lab facilities at Bhaskar Pharmacy College, R.R. District, Hyderabad.

\section{CONFLICT OF INTEREST}

There are no conflicts of interest.

\section{FINANCIAL SUPPORT AND SPONSORSHIP}

Nil.

\section{REFERENCES}

Abdul MH, Raghavendra Rao NG, Srinivasa Rao A. Ileocolonic targeted matrix-mini-tablets of Lornoxicam: In-vivo evaluation. Paper published in in the proceedings of NDCT-2014 conference Organized by Centre for Chemical Sciences and Technology, Institute of Science and
Technology at Jawaharlal Nehru Technological University, Hyderabad on $25^{\text {th }} \& 26^{\text {th }}$ June $2014 ; 301-308$.

Akhgari A, Afrasiabi Garekani H, Sadeghi F, Azimaie M. Statistical optimization of indomethacin pellets coated with $\mathrm{pH}$-dependent methacrylic polymers for possible colonic drug delivery. Int J Pharm. 2005; 305: 22-30.

Aleksandar Aleksovski, Rok Dreu, Mirjana Gašperlin, Odon Planinsek. Mini-tablets: a contemporary system for oral drug delivery in targeted patient groups. Exp Opi Drug Del. 2015; 12(1): 65-84.

Anne Zajicek, Michael Fossler J, Jeffrey Barrett S, Jeffrey Worthington H, Robert Ternik, Georgia Charkoftaki et al. A Report from the Pediatric Formulations Task Force: Perspectives on the State of ChildFriendly Oral Dosage Forms. AAPS J. 2013; 15(4): 1072-1081.

Anupriya Adhikari, Ganesh Bhatt, Preeti Kothiyal A. Chronotherapeutics Approach to the Management of Hypertension: A Review. Ind J Nov Dr del 2014; 6(4): 297-305.

Brabander CD, Vervaet C, Görtz JP, Remon JP, Berlo JA. Bioavalability of Ibuprofen from matrix mini-tablets based on a mixture of starch and microcrystalline wax. Int J Pharm. 2000; 208: 81-86.

Carla Lopes M, Jose Manual Souza Lobo, Jaao Pinto F, Paulo Costa. Compressed mini-tablet as a biphasic drug delivery system. Int $\mathrm{J}$ Pharm. 2006; 323: 93-100.

Deepak Garg, Vipin Saini, Sumeet Gupta, Deepak Kapoor N, Lokesh Kumar Joshi. Oral Disintegrating Minitablets: Mini Review. DHR Int J Pharm Sci. 2013; 4(2): 66-73.

Dey NS, Majumdar S, Rao MEB. Multiparticulate Drug Delivery Systems for Controlled Release. Trop J Pharm Res. 2008; 7(3): 1067-1075.

Drelaywaja. Chronotherapeutics: Right drugs at right time can heal effectively, Healthmad. 2010; March 22. Available at http://healthmad.com.

Evans DF, Pye G, Bramley R, Clark AG, Dyson TJ, Hardcastle JD. Measurement of gastrointestinal $\mathrm{pH}$ profiles in normal ambulant human subjects. Gut. 1988; 29(8): 1035-1041.

Gaber DM, Noha Nafee, Osama Abdallah Y. Mini-tablets versus pellets as promising multiparticulate modified release delivery systems for highly soluble drugs. Int J Pharm. 2015; 488: 86-94.

Gothaskar AV, Joshi AM, Joshi NH. Pulsatile drug delivery system - a review. Drug Del Tech. 2004; 4: 1-14.

Hadi MA, Raghavendra Rao NG, Srinivasa Rao A. Formulation and evaluation of $\mathrm{pH}$ responsive mini-tablets for ileo-colonic targeted drug delivery. Trop J Pharm Res. 2014; 13(7): 1021-1029.

Hadi MA, Raghavendra Rao NG, Srinivasa Rao A. Pharmacokinetic parameters determination and In vitro-In vivo correlation of ileo-colonic targeted $\mathrm{pH}$ responsive coated mini-tablets of Naproxen. Sci Pharm. 83 (4); 2015: 645-658.

Hrashesky WIM. Timing is everything. The Sci. 1994: 32-37.

Hue Vuong, Marina Revina, Rajab R. Siabhoomi. Evaluation of the enteric performance of Lansoprazole Mini-tablets coated in a perforated pan. Poster reprint Amer Ass Pharm Sci. Annual Meeting, Nov, 2008.

Ivanovska V, Rademaker CMA, Dijk van L. Mantel-Teeuwisse AK. Pediatric drug formulations: a review of challenges and progress Pediatrics. 2014; 134(2): 361-372.

Jain D, Pathak D, Pathak K. Pharmaceutical product development technologies based on the biopharmaceutical classification system. Pharmazie. 2009; 64(8): 483-490.

Karthikeyan D, Vijayalaxmi A. Santhosh Kumar C. Formulation and evaluation of biphasic Delivery system of aceclofenac mini-tablets in hard gelatin capsules. Int J nov tre Pharm Sci. 2013; 3(2): 39-45.

Krishainah YSR, Satyanarayan S. Colon-specific drug delivery system. In: Jain NK, editor. Advances in controlled and novel drug delivery. 1st Ed. New Delhi: CBS publishers and distributors. 2001: 89-119.

Laila Fatima, Ali Asghar, Sajeev Chandran. Multiparticulate Formulation Approach to Colon Specific Drug Delivery: Current Perspectives. Pharm Pharmaceut Sci 2006; 9(3): 327-338.

Lennartz P, Mielck JB, Minitabletting: improving the compactibility of paracetamol powder mixtures. Int J Pharm. 1998; 173: 75-85. 
Mastiholimath VS, Dandagi PM, Samata Jain S, Gadad AP and Kulkarni AR. Time and $\mathrm{pH}$ dependent colon specific, pulsatile delivery of theophylline for nocturnal asthma. Int J Pharm. 2007; 328: 49-56.

Leon Lachman, Herbert Lieberman A. The theory and practice of industrial pharmacy. Special Indian edition. 2009: pp. 293-373.

Meena A, Kumar B, Suriya Prakash TNK and Senthamarai R. Development and evaluation of pulsatile drug delivery system of lornoxicam. Int J Pharm Wor Res. 2011; 2(2): 1-15.

Mirelabodea, Ioan tomuta, Sorin leucuta. Film coating preparation of metoprolol tartrate mini-tablets and in vitro drug release studies. Farmacia. 2010; 58(6): 719-727.

Mohd Abdul Hadi, Nidagurthi Guggilla Raghavendra Rao, Avanapu Srinivasa Rao. Matrix-mini-tablets of Lornoxicam for targeting early morning peak symptoms of rheumatoid arthritis. Iran J Bas Med Sci. 2014; 17: 357-369.

Mohd Abdul Hadi, Nidagurthi Guggilla Raghavendra Rao, Avanapu Srinivasa Rao. Formulation and evaluation of mini-tablets-filledpulsincap delivery of Lornoxicam in the chronotherapeutic treatment of rheumatoid arthritis. Pak J Pharm Sci. 2015; 28(1): 185-193.

Mohd Abdul Hadi, Raghavendra Rao NG, Srinivasa Rao A. Formulation and evaluation of ileo-colonic targeted matrix-mini-tablets of Naproxen for chronotherapeutic treatment of rheumatoid arthritis. Saud Pharm J. 2016; 24:64-73.

Mohapatra SK, Kshirsagar SJ, Bhalekar MR, Shukla GN, Patil AV. Development and evaluation of enzymatically triggered multiparticulate colon targeted drug delivery system. Int J Res Ayu Pharm. 2011; 2: 211-215.

Motor Leela Keerthi, Shireesh Kiran R, Uma Maheshwar Rao V, Aparna Sannapu, Avaru Geetha Dutt, Kalakuntla Sai Krishna. Pharmaceutical Mini-Tablets, its Advantages, Formulation Possibilities and General Evaluation Aspects: A Review. Int J Pharm Sci Rev Res. 2014; 28(1): 214-221.

Noorana tehseen, Vinay rao and Mohd abdul hadi. Design and characterization of twice-daily mini-tablets formulation of pregabalin. Int J Pharm Pharm Sci. 2013; 5(1): 168-175.
Sajan J, Cinu TA, Chacko AJ, Litty J, Jaseeda T. Chronotherapeutics and Chronotherapeutic Drug Delivery Systems. Trop J Pharm Res. 2009; 8(5): 467-475.

Schmidt C, Kleinebudde P. Influence of the granulation step on pellets prepared by extrusion/spheronization, Chem Pharm Bull. 1999; 47(3): 405-412.

Sirisha VRK, Vijaya Sri K, Suresh K. Kamalakar Reddy G. Multiple unit pellet systems: A review. Int J Pharm. 2012; 2(2): 419-425.

Sindhu, Abraham and Srinath MS. Development of modified pulsincap drug delivery system of metronidazole for drug targeting. Ind J Pharm Sci. 2007; 69(1): 24-27.

Shivkumar HN, Sarosija Suresh, Desai BG. Design and evaluation of $\mathrm{pH}$ sensitive mini-tablets for chronotherapeutics delivery of theophylline. Ind J Pharm Sci. 2007; 69: 73-79.

Smolensky MH, Peppar AN. Chronobiology drug delivery and chronopharmaceutics. Adv Drug Del 2007;59:825-851.

Sona Chaudhary S, Hetal Patel K, Punit Parejiya B, Pragna Shelat K. Chronomodulated drug delivery system of urapidil for the treatment of hypertension. Int J Pharm Investig. 2015; 5 (2): 107-113.

Suresh H, Pathak S. Chronopharmaceutics. Emerging role of bio-rhythms in optimizing drug therapy. Ind J Pharm Sci 2005; 67(2): 135 140 .

Patel RR, Patel JK. Novel technologies of oral controlled release drug delivery system. Syst Rev Pharm. 2010; 1(2): 128-132.

How to cite this article:

Abdul Hadi M, Rao NGR, Rao AS, Mahtab T, Tabassum S. A Review on Various Formulation Methods in preparing Colon targeted mini-tablets for Chronotherapy. J App Pharm Sci, 2018; 8(03): 158-164. 